\title{
Ancestry-informative markers for African Americans based on the Affymetrix Pan-African genotyping array
}

Genetic admixture has been utilized as a tool for identifying loci associated with complex traits and diseases in recently admixed populations such as African Americans. In particular, admixture mapping is an efficient approach to identifying genetic basis for those complex diseases with substantial racial or ethnic disparities. Though current advances in admixture mapping algorithms may utilize the entire panel of SNPs, providing ancestry-informative markers (AIMs) that can differentiate parental populations and estimate ancestry proportions in an admixed population may particularly benefit admixture mapping in studies of limited samples, help identify unsuitable individuals (e.g., through genotyping the most informative ancestry markers) before starting large genomewide association studies (GWAS), or guide larger scale targeted deep re-sequencing for determining specific disease-causing variants. Defining panels of AlMs based on commercial, high-throughput genotyping platforms will facilitate the utilization of these platforms for simultaneous admixture mapping of complex traits and diseases, in addition to conventional GWAS. Here, we describe AIMs detected based on the Shannon Information Content (SIC) or $\mathrm{F}_{\mathrm{st}}$ for African Americans with genome-wide coverage that were selected from $\sim 2.3$ million single nucleotide polymorphisms (SNPs) covered by the Affymetrix Axiom Pan-African array, a newly developed genotyping platform optimized for individuals of African ancestry. 


\section{Affymetrix Pan-African Genotyping Array}

4

5

6

7

8

15

\section{*Correspondence to:}

Wei Zhang, Ph.D., 840 S. Wood St., 1200 CSB (M/C 856), Chicago, IL 60612, USA; Tel: +1 (312) 413-2024; E-mail: weizhang.chicago@gmail.com

Running Title: AIMs for African Americans

\section{Abstract}


45 Genetic admixture has been utilized as a tool for identifying loci associated with complex traits

46 and diseases in recently admixed populations such as African Americans. In particular, admixture

47 mapping is an efficient approach to identifying genetic basis for those complex diseases with

48 substantial racial or ethnic disparities. Though current advances in admixture mapping algorithms

49 may utilize the entire panel of SNPs, providing ancestry-informative markers (AIMs) that can

50 differentiate parental populations and estimate ancestry proportions in an admixed population

51 may particularly benefit admixture mapping in studies of limited samples, help identify

52 unsuitable individuals (e.g., through genotyping the most informative ancestry markers) before

53 starting large genome-wide association studies (GWAS), or guide larger scale targeted deep re-

54 sequencing for determining specific disease-causing variants. Defining panels of AIMs based on

55 commercial, high-throughput genotyping platforms will facilitate the utilization of these

56 platforms for simultaneous admixture mapping of complex traits and diseases, in addition to

57 conventional GWAS. Here, we describe AIMs detected based on the Shannon Information

58 Content (SIC) or $\mathrm{F}_{\mathrm{st}}$ for African Americans with genome-wide coverage that were selected from

$59 \sim 2.3$ million single nucleotide polymorphisms (SNPs) covered by the Affymetrix Axiom Pan-

60 African array, a newly developed genotyping platform optimized for individuals of African

61 ancestry.

62

63

64

65

66

67

68

69

Introduction

High throughput genotyping arrays have facilitated genome-wide association studies

70 (GWAS) on complex traits (Hindorff et al. 2009) including risks for common, complex diseases 
71 and drug response. In contrast to a conventional GWAS in a homogeneous parental populations

72 (e.g., Caucasians), admixture mapping or mapping by admixture linkage disequilibrium (MALD)

73 has begun to be demonstrated as a powerful tool for identifying disease-causing genetic variants

74 in recently admixed populations, such as African Americans that have both West African and

75 European American ancestry (McKeigue 2005). For example, recent admixture mapping studies

76 have identified loci associated with disease risks such as prostate cancer (Ricks-Santi et al. 2012),

77 lung cancer (Schwartz et al. 2011), and traits like blood pressure/obesity (Shetty et al. 2012) in

78 African Americans. Admixture mapping assumes that near a disease causing genetic variant there

79 will be enhanced ancestry from the population that has greater risk of getting the disease

80 (Patterson et al. 2004). Therefore, by calculating the proportion of ancestry along the genome,

81 one could use that information to identify disease causing loci in an admixed population with low

82 resolution. Subsequent fine mapping restricted to the identified genomic regions may greatly

83 increase the power of the study.

85 It has been demonstrated that 1,500-2,500 ancestry-informative markers (AIMs) with

86 genome-wide coverage would be sufficient (Winkler et al. 2010) to identify the ancestral

87 chromosome segments for recently admixed populations. To leverage on the power of admixture

88 mapping in African American for identifying disease causing genetic variants that may explain

89 health disparities between populations, panels of AIMs have been proposed for commercially-

90 available high throughput genotyping arrays including the Affymetrix SNP 6.0 and Illumina 1M

91 (Chen et al. 2010; Tandon et al. 2011). These genotyping arrays however are likely biased to

92 genetic variations detected from Caucasian samples. The Affymetrix Pan-African array, which

93 interrogates approximately 2.3 million SNPs, was designed for a much greater coverage of

94 genetic variations in African individuals. A panel of AIMs based on the Pan-African array may

95 enhance the distinguishing of parental populations as well as improve genome coverage. Recent 
96 advances in statistical genetics have begun to allow admixture mapping utilizing the entire panel

97 of genotyped SNPs (Baran et al. 2012; Churchhouse \& Marchini 2013; Maples et al. 2013),

98 however, we reasoned that providing a panel of AIMs may particularly benefit studies of a

99 limited sample size, help identify unsuitable individuals by genotyping the most informative

100 markers before starting a large GWAS, or guide larger scale targeted re-sequencing projects to

101 pinpoint causal variants. We describe here AIMs identified for the Affymetrix Pan-African array

102 based on Shannon Information Content (SIC) or $\mathrm{F}_{\mathrm{st}}$ for African Americans using the 1000

103 Genomes Project (Abecasis et al. 2010) data as references for parental populations.

105 Materials and Methods

106 SNPs covered on the Pan-African array

107 The Affymetrix Axiom Genome-Wide Pan AFR Genotyping platform (Pan-African array)

108 (Affymetrix, Inc., Santa Clara, California) covers $\sim 2.3$ million SNPs optimized for individuals of

109 African ancestry. The Pan-African array was designed to offer $\geq 90 \%$ coverage of SNPs on the

110 Yoruba genome with minor allele frequency (MAF) greater than $2 \%$. Annotations for the Pan-

111 African array can be accessed at the Affymetrix website (http://www.affymetrix.com/). As a

112 platform optimized for individuals of African individuals, the Pan-African array has been

113 extensively validated in African populations from the HapMap Project (Altshuler et al. 2010),

114 including the Luhya from western Kenya (LWK), Maasai from eastern Kenya (MWK), Yoruba

115 from Ibadan, Nigeria (YRI), and the African Ancestry in the Southwest USA (ASW) (Lu et al.

116 2011). This platform offers high genomic coverage ( $>85 \%)$ in admixed populations with West

117 African ancestry, thus particularly suitable for genome-wide scans in African American

118 individuals (admixture of African and European populations).

119 Obtaining allele frequency and genetic map distances on parental populations 
120 Genotypes for 2176716 SNPs covered by the Pan-African array were extracted from the 1000

121 Genomes Project (Abecasis et al. 2010) Phase I data for the 85 CEU (Caucasian residents from

122 Utah, USA) and 88 YRI unrelated samples, representing the two major parental populations for

123 African Americans (Western Africans and Europeans). Genome-wide genetic map distances of

124 SNPs for genome assembly GRCh37 (Frazer et al. 2007) were downloaded from the website

125 (http://bochet.gcc.biostat.washington.edu/beagle/genetic_maps).

\section{Selection of ancestry-informative markers}

127 We aimed to pick the SNPs that were expected to provide the highest mutual information

128 content to ancestry or fixation index (i.e., $\mathrm{F}_{\text {st }}$, a measure of population differentiation due to

129 genetic structure) in the genome using an iterative procedure, conditional on the observed allele

130 frequencies in the 1000 Genomes Project CEU and YRI samples.

131 (a) Calculation of mutual information content: Allele frequencies for the CEU and YRI samples

132 were used to calculate the Shannon Information Content (SIC) for each SNP using a formula

133 from previous studies (Smith et al. 2004; Tandon et al. 2011),

$$
\mathrm{SIC}=-\sum_{i=0}^{1}\left(a_{i 0}+a_{i 1}\right) \ln \left(a_{i 0}+a_{i 1}\right)-\sum_{j=0}^{1}\left(a_{0 j}+a_{1 j}\right) \ln \left(a_{0 j}+a_{1 j}\right)+\sum_{i=0}^{1} \sum_{j=0}^{1} a_{\mathrm{ij}} \ln \left(a_{\mathrm{ij}}\right)
$$

135 , where $a_{00}=(1-m) \times p^{\mathrm{YRI}}, \quad a_{01}=\left(m \times p^{\mathrm{CEU}}\right), \quad a_{10}=(1-m) \times\left(1-p^{\mathrm{YRI}}\right)$, and

$136 a_{11}=m \times\left(1-p^{\mathrm{CEU}}\right)$. Here, $p^{C E U}$ and $p^{Y R I}$ are the allele frequencies in the CEU (European) and

137 YRI (African) samples, and $m$ is the proportion of European ancestry in African Americans,

138 which was set to 0.20 following the same assumption of $20 \%$ European ancestry (Tandon et al.

139 2011). Notably, SNP selection was found not very sensitive to the choice of m (Smith et al.

140 2004). In addition, the $F_{\text {st }}$ was also computed for each of the 2176716 SNPs between the two

141 parental populations based on Wright's approximate formula (Wright 1950),

$142 \quad F_{\mathrm{ST}}=\left(H_{T}-H_{S}\right) / H_{T}$ 


\section{PeerJ Reviewing Manuscript}

143 , where $H_{T}$ represents expected heterozygosity per locus of the total population and $H_{S}$ represents

144 expected heterozygosity of a subpopulation.

145 (b) Selection of AIMs: We aimed to detect AIMs that are not packed around certain genomic

146 regions due to linkage disequilibrium (LD), thus being more representative of the genome. Since

147 LD declines gradually with increased genetic distance (Shifman et al. 2003), we assume each

148 SNP is not in LD with distant SNPs more than $0.25 \mathrm{cM}(\sim 250 \mathrm{~kb})$ away, similar to what was used

149 in previous publications (Tandon et al. 2011). We selected AIMs using an iterative procedure for

150 each chromosome: 1) SNPs were ranked based on SIC; 2) SNP with the highest SIC was selected

151 as a candidate AIM; 3) Any SNPs within $0.25 \mathrm{cM}$ or within $250 \mathrm{~kb}$ of the selected SNP were

152 excluded; 4) Steps 2 and 3 were repeated until no more SNPs left. To avoid densely packed

153 markers, no more than 8 candidate AIMs were selected within any $4 \mathrm{cM}$ region. This procedure

154 ensured a good coverage of AIMs across the entire genome. The quality of the detected candidate

155 AIMs was examined using the build-in data quality checking procedure of ANCESTRYMAP 2.0

156 (Patterson et al. 2004) for extracting top "bad" markers, for which allele counts for the ancestral

157 (African and European) genotypes appeared to be grossly inconsistent with counts on the 56

158 unrelated ASW samples from 1000 Genome Project (Abecasis et al. 2010). After applying the

159 ANCESTRYMAP quality checks, we obtained the final panel of AIMs. We also repeated the

160 same selection procedure using $\mathrm{F}_{\mathrm{st}}$ to identify a companion panel of AIMs. Supplemental Tables 1

161 and 2 contain detailed information on the final AIMs.

162 Evaluation of the detected AIMs for the Pan-African array

163 The informativeness of the AIMs was evaluated at each SNP using the ANCESTRYMAP-

164 generated rpower value, which is a measure of uncertainty in ancestry inference at a given locus.

165 Specifically, rpower is the expected value of the squared correlation between inferred and true

166 ancestry (Patterson et al. 2004). In addition, proportion of variance explained (PVE) by the first

167 principal component (PC) using the detected AIMs on the CEU, YRI, and ASW samples was 
168

169

170

171

172

173

174

175

176

177

178

179

180

181

182

183

184

185

186

187

188

189

190

191

192 compared with PVE's from previously published AIMs (based on Affymetrix SNP 6.0 and Illumina 1M arrays) (Tandon et al. 2011) as well as 1000 random sets of SNPs.

\section{Results and Discussion}

Given that the Pan-African array was population-optimized, this platform is expected to offer higher coverage of genetic variation for individuals of African ancestry than previous platforms mostly designed based on Caucasians. Genotyping using the Affymetrix Pan-African array will provide opportunities for performing admixture mapping in African Americans to detect genetic variants associated with those traits that exhibit disparities between parental populations, for instance certain cancers (Schwartz et al. 2011). The primary result from this study was a panel of SNPs based on the Pan-African array. We acknowledge that with recent advances in statistical genetics, admixture mapping in African Americans may not rely on a limited number of AIMs any more (Baran et al. 2012; Churchhouse \& Marchini 2013; Maples et al. 2013). We propose that some applications for our detected AIMs could include: 1) to facilitate admixture mapping in limited samples; 2) to help identify problematic individuals through genotyping some top-ranking AIMs before starting a large GWAS; 3 ) to guide targeted resequencing projects that may not have genome-wide genotypic data.

Using an iterative selection algorithm, a total of 6011 candidate AIMs were detected based on SIC, which can measure the uncertainty in genome-wide ancestry or ancestry at a given locus (Tandon et al. 2011). We further examined the quality of these candidates using the build-in checking procedure of ANCESTRYMAP (Patterson et al. 2004) and identified a final set of AIMs with 5995 SNPs based on SIC. We also repeated the same analysis using F $_{\text {st }}$ to identify a companion panel of 6012 after ANCESTRYMAP checking from 6034detected candidate SNPs. 
193 The selected AIMs with rs numbers, genomic positions, reference alleles, alternative alleles, and

194 allele counts in the CEU or YRI samples are shown in supplemental materials. Overall, AIMs

195 based on SIC and $\mathrm{F}_{\text {st }}$ performed consistently with each other. The average rpower (i.e., average

196 ancestry information) of the AIMs based on SIC or $F_{\text {st }}$ was 0.85 (Figure 1A), compared to $\sim 0.81$

197 for previous AIMs detected for Affymetrix SNP 6.0 and Illumina 1M arrays (Tandon et al. 2011).

198 The average proportion of European ancestry in ASW was estimated to be 0.25 and 0.24 and the

199 average generations of admixture was estimated to be 5.4 and 5.5 using the AIMs based on SIC

200 and $\mathrm{F}_{\text {st }}$, respectively, consistent with previous estimation (Tandon et al. 2011).

201 The availability of dense genetic variation data from the HapMap Project (HapMap 2003;

202 HapMap 2005) allows a genome-wide analysis of population differentiation. In particular, the

203 CEU (European) and YRI (African) samples represented the two major parental populations of

204 African Americans. Our major criteria of identifying AIMs were designed 1) to enrich SNPs with

205 higher information content (or $\mathrm{F}_{\text {st }}$ ) between the CEU and YRI samples; and 2) to have a

206 comprehensive genomic coverage. The genome-wide iterative scan for AIMs based on a genetic

207 distance bin in a size of $0.25 \mathrm{cM}$, guaranteed a comprehensive coverage of the entire human

208 genome, as well as limit the possibility that the identified AIMs are in strong LD in a particular

209 genomic region, as described in previous publications (Chen et al. 2010; Tandon et al. 2011). The

210 final AIMs are those SNPs with the highest SIC (or $\mathrm{F}_{\text {st }}$ ) separated by at least the distance of 0.25

$211 \mathrm{cM}(\sim 250 \mathrm{~kb})$ between the two parental populations. the detected AIMs were able to recapture the

212 most prominent population structures by being tested on the combined HapMap CEU, YRI, and

213 ASW samples (Figure 1B). A simulation analysis demonstrated that the detected AIMs based on

214 the Pan-African array explained substantially higher proportion of variance by the first PCs in the

215 same population than random sets of SNPs in the human genome (Figure 1C). Though our

216 analysis showed that the AIMs detected based on SIC and $\mathrm{F}_{\text {st }}$ performed consistently, given some 


\section{PeerJ Reviewing Manuscript}

217 potential problems of $\mathrm{F}_{\mathrm{st}}$, in particular its dependency on within-population diversity (Sherwin

218 2010), we generally recommend the use of the final panel of AIMs detected based on SIC.

219 The assumption of no LD based on $0.25 \mathrm{cM}(\sim 250 \mathrm{~kb})$ could be stringent and cause loss

220 of some informative SNPs, given that the average distance of LD decay between SNP pairs is

221 around 20-30 kb across diverse populations, with generally shorter distance in African Americans

222 (Shifman et al. 2003). Nevertheless, this cutoff was chosen to balance between minimizing the

223 possibility of LD and the comprehensive genomic coverage of AIMs (Tandon et al. 2011).

224 In summary, the Affymetrix Pan-African array provides a population-optimized

225 genotyping platform for GWAS in individuals of African ancestry. The genotypic data profiled by

226 this platform also offers opportunities for admixture mapping in African Americans, a recently

227 admixed population, for certain complex traits and disease susceptibilities with disparities

228 between parental populations. The AIMs we described in this study represent the most

229 informative sets of unlinked markers that can be an important resource to facilitate such

230 applications based on this new tool.

231

232

References

233

Abecasis GR, Altshuler D, Auton A, Brooks LD, Durbin RM, Gibbs RA, Hurles ME, and McVean GA. 2010. A map of human genome variation from population-scale sequencing. Nature 467:1061-1073.

Altshuler DM, Gibbs RA, Peltonen L, Altshuler DM, Gibbs RA, Peltonen L, Dermitzakis E, Schaffner SF, Yu F, Peltonen L, Dermitzakis E, Bonnen PE, Altshuler DM, Gibbs RA, de Bakker PI, Deloukas P, Gabriel SB, Gwilliam R, Hunt S, Inouye M, Jia X, Palotie A, Parkin M, Whittaker P, Yu F, Chang K, Hawes A, Lewis LR, Ren Y, Wheeler D, Gibbs RA, Muzny DM, Barnes C, Darvishi K, Hurles M, Korn JM, Kristiansson K, Lee C, McCarrol SA, Nemesh J, Dermitzakis E, Keinan A, Montgomery SB, Pollack S, Price AL, Soranzo N, Bonnen PE, Gibbs RA, Gonzaga-Jauregui C, Keinan A, Price AL, Yu F, Anttila V, Brodeur W, Daly MJ, Leslie S, McVean G, Moutsianas L, Nguyen H, Schaffner SF, Zhang Q, Ghori MJ, McGinnis R, McLaren W, Pollack S, Price AL, Schaffner SF, Takeuchi F, Grossman SR, Shlyakhter I, Hostetter EB, Sabeti PC, Adebamowo CA, Foster MW, Gordon DR, Licinio J, Manca MC, Marshall PA, Matsuda I, Ngare D, Wang VO, Reddy D, Rotimi CN, Royal CD, Sharp RR, Zeng C, Brooks LD, and McEwen JE. 2010. Integrating common and rare genetic variation in diverse human populations. Nature 467:52-58. 
Baran Y, Pasaniuc B, Sankararaman S, Torgerson DG, Gignoux C, Eng C, Rodriguez-Cintron W, Chapela R, Ford JG, Avila PC, Rodriguez-Santana J, Burchard EG, and Halperin E. 2012. Fast and accurate inference of local ancestry in Latino populations. Bioinformatics 28:1359-1367.

Chen G, Shriner D, Zhou J, Doumatey A, Huang H, Gerry NP, Herbert A, Christman MF, Chen Y, Dunston GM, Faruque MU, Rotimi CN, and Adeyemo A. 2010. Development of admixture mapping panels for African Americans from commercial high-density SNP arrays. BMC Genomics 11:417.

Churchhouse C, and Marchini J. 2013. Multiway admixture deconvolution using phased or unphased ancestral panels. Genet Epidemiol 37:1-12.

Frazer KA, Ballinger DG, Cox DR, Hinds DA, Stuve LL, Gibbs RA, Belmont JW, Boudreau A, Hardenbol P, Leal SM, Pasternak S, Wheeler DA, Willis TD, Yu F, Yang H, Zeng C, Gao Y, Hu H, Hu W, Li C, Lin W, Liu S, Pan H, Tang X, Wang J, Wang W, Yu J, Zhang B, Zhang Q, Zhao H, Zhao H, Zhou J, Gabriel SB, Barry R, Blumenstiel B, Camargo A, Defelice M, Faggart M, Goyette M, Gupta S, Moore J, Nguyen H, Onofrio RC, Parkin M, Roy J, Stahl E, Winchester E, Ziaugra L, Altshuler D, Shen Y, Yao Z, Huang W, Chu X, He Y, Jin L, Liu Y, Shen Y, Sun W, Wang H, Wang Y, Wang Y, Xiong X, Xu L, Waye MM, Tsui SK, Xue H, Wong JT, Galver LM, Fan JB, Gunderson K, Murray SS, Oliphant AR, Chee MS, Montpetit A, Chagnon F, Ferretti V, Leboeuf M, Olivier JF, Phillips MS, Roumy S, Sallee C, Verner A, Hudson TJ, Kwok PY, Cai D, Koboldt DC, Miller RD, Pawlikowska L, Taillon-Miller P, Xiao M, Tsui LC, Mak W, Song YQ, Tam PK, Nakamura Y, Kawaguchi T, Kitamoto T, Morizono T, Nagashima A, Ohnishi Y, Sekine A, Tanaka T, Tsunoda T, Deloukas P, Bird CP, Delgado M, Dermitzakis ET, Gwilliam R, Hunt S, Morrison J, Powell D, Stranger BE, Whittaker P, Bentley DR, Daly MJ, de Bakker PI, Barrett J, Chretien YR, Maller J, McCarroll S, Patterson N, Pe'er I, Price A, Purcell S, Richter DJ, Sabeti P, Saxena R, Schaffner SF, Sham PC, Varilly P, Altshuler D, Stein LD, Krishnan L, Smith AV, Tello-Ruiz MK, Thorisson GA, Chakravarti A, Chen PE, Cutler DJ, Kashuk CS, Lin S, Abecasis GR, Guan W, Li Y, Munro HM, Qin ZS, Thomas DJ, McVean G, Auton A, Bottolo L, Cardin N, Eyheramendy S, Freeman C, Marchini J, Myers S, Spencer C, Stephens M, Donnelly P, Cardon LR, Clarke G, Evans DM, Morris AP, Weir BS, Tsunoda T, Mullikin JC, Sherry ST, Feolo M, Skol A, Zhang H, Zeng C, Zhao H, Matsuda I, Fukushima Y, Macer DR, Suda E, Rotimi CN, Adebamowo CA, Ajayi I, Aniagwu T, Marshall PA, Nkwodimmah C, Royal CD, Leppert MF, Dixon M, Peiffer A, Qiu R, Kent A, Kato K, Niikawa N, Adewole IF, Knoppers BM, Foster MW, Clayton EW, Watkin J, Gibbs RA, Belmont JW, Muzny D, Nazareth L, Sodergren E, Weinstock GM, Wheeler DA, Yakub I, Gabriel SB, Onofrio RC, Richter DJ, Ziaugra L, Birren BW, Daly MJ, Altshuler D, Wilson RK, Fulton LL, Rogers J, Burton J, Carter NP, Clee CM, Griffiths M, Jones MC, McLay K, Plumb RW, Ross MT, Sims SK, Willey DL, Chen Z, Han H, Kang L, Godbout M, Wallenburg JC, L'Archeveque P, Bellemare G, Saeki K, Wang H, An D, Fu H, Li Q, Wang Z, Wang R, Holden AL, Brooks LD, McEwen JE, Guyer MS, Wang VO, Peterson JL, Shi M, Spiegel J, Sung LM, Zacharia LF, Collins FS, Kennedy K, Jamieson R, and Stewart J. 2007. A second generation human haplotype map of over 3.1 million SNPs. Nature 449:851-861.

HapMap. 2003. The International HapMap Project. Nature 426:789-796.

HapMap. 2005. A haplotype map of the human genome. Nature 437:1299-1320.

Hindorff LA, Sethupathy P, Junkins HA, Ramos EM, Mehta JP, Collins FS, and Manolio TA. 2009. Potential etiologic and functional implications of genome-wide association loci for human diseases and traits. Proc Natl Acad Sci U S A 106:9362-9367. 
Lu Y, Patterson N, Zhan Y, Mallick S, and Reich D. 2011. Technical design document for a SNP array that is optimized for population genetics.ftp://ftp.cephb.fr/hgdp_supp10/.

Maples BK, Gravel S, Kenny EE, and Bustamante CD. 2013. RFMix: a discriminative modeling approach for rapid and robust local-ancestry inference. Am J Hum Genet 93:278-288.

McKeigue PM. 2005. Prospects for admixture mapping of complex traits. Am J Hum Genet 76:17.

Patterson N, Hattangadi N, Lane B, Lohmueller KE, Hafler DA, Oksenberg JR, Hauser SL, Smith MW, O'Brien SJ, Altshuler D, Daly MJ, and Reich D. 2004. Methods for highdensity admixture mapping of disease genes. Am J Hum Genet 74:979-1000.

Ricks-Santi LJ, Apprey V, Mason T, Wilson B, Abbas M, Hernandez W, Hooker S, Doura M, Bonney G, Dunston G, Kittles R, and Ahaghotu C. 2012. Identification of genetic risk associated with prostate cancer using ancestry informative markers. Prostate Cancer Prostatic Dis 15:359-364.

Schwartz AG, Wenzlaff AS, Bock CH, Ruterbusch JJ, Chen W, Cote ML, Artis AS, Van Dyke AL, Land SJ, Harris CC, Pine SR, Spitz MR, Amos CI, Levin AM, and McKeigue PM. 2011. Admixture mapping of lung cancer in 1812 African-Americans. Carcinogenesis 32:312-317.

Sherwin W. 2010. Entropy and information approaches to genetic diversity and its expression: genomic geography. Entropy 12:1765-1798.

Shetty PB, Tang H, Tayo BO, Morrison AC, Hanis CL, Rao DC, Young JH, Fox ER, Boerwinkle E, Cooper RS, Risch NJ, and Zhu X. 2012. Variants in CXADR and F2RL1 are associated with blood pressure and obesity in African-Americans in regions identified through admixture mapping. J Hypertens 30:1970-1976.

Shifman S, Kuypers J, Kokoris M, Yakir B, and Darvasi A. 2003. Linkage disequilibrium patterns of the human genome across populations. Hum Mol Genet 12:771-776.

Smith MW, Patterson N, Lautenberger JA, Truelove AL, McDonald GJ, Waliszewska A, Kessing BD, Malasky MJ, Scafe C, Le E, De Jager PL, Mignault AA, Yi Z, De The G, Essex M, Sankale JL, Moore JH, Poku K, Phair JP, Goedert JJ, Vlahov D, Williams SM, Tishkoff SA, Winkler CA, De La Vega FM, Woodage T, Sninsky JJ, Hafler DA, Altshuler D, Gilbert DA, O'Brien SJ, and Reich D. 2004. A high-density admixture map for disease gene discovery in african americans. Am J Hum Genet 74:1001-1013.

Tandon A, Patterson N, and Reich D. 2011. Ancestry informative marker panels for African Americans based on subsets of commercially available SNP arrays. Genet Epidemiol 35:80-83.

Winkler CA, Nelson GW, and Smith MW. 2010. Admixture mapping comes of age. Annu Rev Genomics Hum Genet 11:65-89.

Wright S. 1950. Genetical structure of populations. Nature 166:247-249. 


\section{Figure 1}

Evaluation anlaysis of ancestry-informative markers.

(A) The rpower distributions for AIMs selected based on SIC and $\mathrm{F}_{\mathrm{st}}$. The average rpower is 0.85 ( $s d=0.06$ ) for both lists. (B) Principal components analysis on the 1000 Genomes Project CEU, YRI and ASW panels ( $n=85$ 88, 56 unrelated samples, respectively) using the AIMs detected based on SIC. (C) Comparison of the proportion of variance explained (PVE) by the first PCs derived from the CEU, YRI, and ASW samples. The histogram shows the distribution from 1000 randomly-sampled sets of SNPs according to the number of AIMs (based on SIC) on each chromosome. Circles denote real PVE observations for each panel of AIMs: AIMs selected by SIC (5885 SNPs) and $\mathrm{F}_{\mathrm{st}}$ (6012 SNPs) from Pan-African array, AIMs selected from Affymetrix SNP 6.0 (4290 SNPs), and Illumina 1M (4285 SNPs), respectively. 

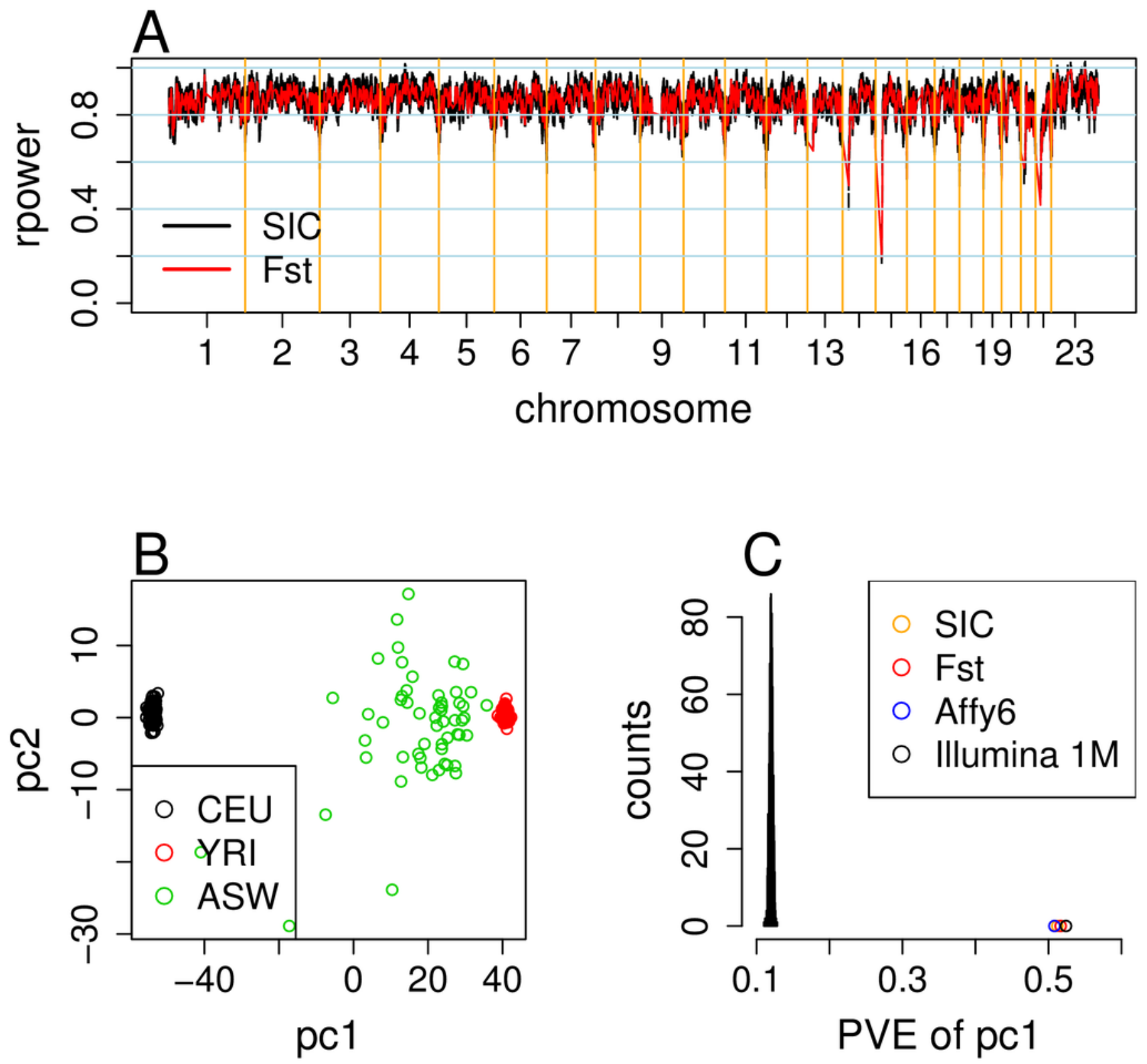